\title{
Novel Hybrid Orthogonal Large Set Code Sequence for High Density Wireless Networks
}

\author{
Nyoman Pramaita ${ }^{1}$ and P. L. Johnson ${ }^{1}$
}

\begin{abstract}
CDMA technique has been successfully employed to implement high capacity mobile networks with optimum channel bandwidth utilization. The two most coveted criteria for the spreading codes used in such network are, the number of codes in the set and the code orthogonality, in order to ensure accommodation of large number of users and as well as minimal interference between the users. Walsh code has proved to be a popular orthogonal code, whereas m-sequence, Gold code and Kasami code have established themselves as popular non-orthogonal binary codes. Small set Orthogonal Kasami code has been proposed and evaluated by researchers for CDMA applications. In this paper the authors report the design of hybrid orthogonal large set (HOLS) code for synchronous CDMA systems. The HOLS code was evaluated in a synchronous CDMA system over different channel conditions. The authors also demonstrate that the BER performance of the HOLS code is comparable to that of the existing orthogonal codes, but outperforms Gold and large set Kasami code sequences. The HOLS code sequence is also shown to outperform the existing code sequences in the number of cells it can support. In terms of multiple access interference (MAI), the cross-correlation function (CCF) of the HOLS code sequence is comparable to that of Walsh and orthogonal small set Kasami codes, and much better than those of Gold and large set Kasami code sequences. HOLS code sequence has comparable auto-correlation function (ACF) with that of Gold, large set Kasami and orthogonal small set Kasami code sequences and a much better ACF than that of Walsh code sequence.
\end{abstract}

Keywords—BER, Large set Kasami Code, Mobile Network, SNR, Spreading Codes.

\section{INTRODUCTION}

$\mathrm{C}$ ode division multiple access (CDMA) system is one of the applications based on spread spectrum requiring a unique spreading code sequence for each user [1]-[3]. In the downlink of CDMA mobile network, a signal coming from a base station has a component intended for a desired user and components intended for other users in the same cell using the same frequency. From the perspective of the desired user, the components intended for other users are known as multiple access interference (MAI) [4], [5]. The performance of CDMA based wireless system is mainly limited by multiple access interference (MAI) arising due to crosscorrelation between the spreading sequences assigned to different users under multiple access interference limited channel conditions [6].

Binary spreading codes are categorized into orthogonal and non-orthogonal codes. Walsh code has proven its existence as a popular orthogonal code, whereas Gold code, Kasami code, and maximal or m-sequences (sequences having period not exceeding $2^{\wedge} \mathrm{n}-1$ produced by a given linear $n$-stage shift register), have established themselves as popular non-orthogonal binary codes [7]-[9]. One of the primary objectives of the mobile system designers is to create the spreading code with a large family to support large number of users while maintaining low cross-correlation values between them in order to mitigate the effect of MAI [10].

Among the spreading code sequences mentioned above, large set Kasami code sequence has the largest number of spreading codes [11]. It means that this code sequence can accommodate the largest number of users in CDMA or multi-user applications. However, they

\footnotetext{
${ }^{1}$ Nyoman Pramaita and P. L. Johnson are with School of Engineering, Liverpool John Moores University, Liverpool, UK. Email: N.Pramaita@2011.ljmu.ac.uk; P.Johnson@1jmu.ac.uk.
}

have the largest cross-correlation values, almost same as the Gold codes. The larger the cross-correlation values of spreading codes, larger the contribution of the adjacent users to the effect of MAI.

In this paper, the authors propose the design of HOLS code sequence (which is a specific combination of $\mathrm{m}$ sequence and large set Kasami code sequences) that minimizes the effect of MAI and can accommodate largest number of devices compared to any of the existing spreading codes.

The simulation results show that the proposed HOLS code sequence has a BER performance similar to those of Walsh and orthogonal small set Kasami code sequences and superior to those of Gold and large set Kasami code sequences for both flat and frequency selective fading channel conditions. In fact, the capacity of the HOLS code sequence is significantly higher than that of any existing code sequence. In terms of MAI, cross-correlation function (CCF) of the HOLS code sequence is comparable to those of Walsh and orthogonal small set Kasami code sequences and much better than those of Gold and large set Kasami code sequences. The proposed HOLS code sequence has comparable auto-correlation function (ACF) with that of Gold, large set Kasami and orthogonal small set Kasami code sequences and a much better ACF than that of Walsh code sequence.

\section{METHOD}

In this section, we propose the method to generate the proposed HOLS code sequence and evaluate it through a range of simulations using Matlab package. This evaluation includes BER performance of the HOLS code sequence under various channel conditions in synchronous CDMA system and compared to a range of non-orthogonal, Walsh, and orthogonal small set Kasami code sequences. Also, CCF and ACF of the code sequences was evaluated. 


\section{A. The HOLS Code Generation}

Non-orthogonal small set and large set Kasami code sequences are obtained by decimation of $m$-sequence. However, conversion of non-orthogonal large set Kasami code sequence into HOLS code sequence is carried out by following a certain combination of $m$-sequence and non-orthogonal large set Kasami code sequence, as shown in Figure 1.

The simulation of the code was carried out using Matlab programming. The number of codes that can be obtained by the algorithms is given by:

$M=$ (Number of groups) . (Number of members in a group)

Where, number of groups $=\left(N \frac{n}{2}\right)+(2 n(n-1))$

and number of members in a group $=(N+1)$

$N$ and $n$ in equations (2) and (3) are the length of the non-orthogonal codes and the number of shift registers respectively.

A set of codes is said to be orthogonal, when the crosscorrelation between any two members of the same code set is zero. However, this does not mean that any member in a group of codes should also be orthogonal to all the members in other groups. In a synchronous CDMA system this ensures that there is no interference between signals transmitted by the same station.

In this context, the proposed code set is proven to be orthogonal from the cross-correlation values between any two members of the code set, which is zero as shown in Figure 20. For $n=6$, referring to equation (2) there are 249 code groups where each code group has 64 members. One group in this family of codes relate to one cell in a CDMA system. So, the proposed code sequence can be distributed among the 249 cells.

\section{B. Simulation of the HOLS code sequence}

The code sequences considered in this paper were implemented in a synchronous CDMA system, via simulation using Matlab programming. The BER performance of the HOLS code sequence was evaluated.

A set of assumptions have been made for the simulation of the system, which are given in section 2 . The block diagram and the rationale behind the blocks used to simulate the synchronous CDMA system are given in section 1. Number of Monte Carlo simulations used for this system is five times.

1) Block diagram of the proposed model

The block diagram of the simulation model used is shown in Figure 2. This simulation is performed under different channel conditions, namely Rayleigh flat fading, and Rayleigh frequency selective fading.

Binary digits (bits) information was generated using random function generator and modulated using DBPSK modulator to produce DBPSK symbols. The CDMA system assigns each user with different spreading sequences from the same set of sequences to spread the DBPSK symbols. The spread spectrum signal is then transmitted through the channel. At the channel output, the received signal is correlated with the intended user's spreading code using a correlation receiver. This process will produce the desired signal and as well as the interfering signals from other users (interfering users). These interfering signals will be rejected by a despreading process. The desired signal is then demodulated to obtain desired bit information.
2) Assumptions for the simulation of the synchronous CDMA system

Based on the block diagram of the simulation model shown in Fig. 2, the assumptions for the simulations are as follows:

a. The input considered is Bernoulli distribution with same probability between bit 0 and 1 .

b. The number of bits for the simulation is 300.000 bits.

c. Bit rate $=1000 \mathrm{bits} / \mathrm{sec}$ per user.

d. Modulation is DBPSK.

e. The number of users is 64 except for orthogonal small set Kasami code sequence which is 63 users since it is the maximum number of codes in a group

f. The power transmitted by each user's signal at the base station is 1 Watt.

g. Spreading code lengths of 63 and 64 chips are considered for the non-orthogonal and orthogonal code sequences respectively.

$\mathrm{h}$. The receiver is a correlation receiver.

i. The desired signal and MAI signals are chip synchronized at the receiver.

j. Each signal is assumed to arrive at the receiver with equal average power.

$\mathrm{k}$. There is no interference from other cells.

1. Flat fading channel is modeled by one path with a path gain equal to $0 \mathrm{~dB}$ and a Doppler shift of 30 $\mathrm{Hz}$.

m. Frequency selective fading channel is modeled with four path time delays $(0,1$ chip duration, 2 chips duration, 3 chips duration), four path gains (0 $\mathrm{dB}$,

n. $-3 \mathrm{~dB},-6 \mathrm{~dB}-9 \mathrm{~dB}$ ), and with $30 \mathrm{~Hz}$ of Doppler shift.

\section{RESULT AND DISCUSSION}

Evaluation of the HOLS code sequence was carried out through a range of simulations using Matlab software package.

\section{A. BER of HOLS code sequence under Rayleigh flat} fading channel condition

As seen from Figure. 3, the proposed HOLS code sequence has similar BER performance to that of Walsh and orthogonal small set Kasami code sequence, but outperforms the non-orthogonal code sequences, namely, Gold and large set Kasami code in synchronous CDMA system under Rayleigh flat fading channel. The BER performance of the large set Kasami code sequence is better than that of the Gold code sequence under this channel condition. The reasons are discussed in the following paragraphs.

As seen in Figure 4, the proposed HOLS, Walsh and orthogonal small set Kasami code sequences have consistently superior BER performance for increasing number of users sharing the same flat fading channel for an SNR value of $30 \mathrm{~dB}$. This implies that under this channel condition, the orthogonal code sequences do not suffer from interference from other users using the spreading code sequences from the same set of code sequences and hence, the receiver has only to cope with the noise introduced by the channel. So, the orthogonal code sequences have similar signal levels at specific 
sampling interval as illustrated by their eye diagrams in Figure 4, 5 and 6 . Therefore, the orthogonal code sequences have similar BER performance in synchronous CDMA system under flat fading channel.

Large set Kasami code sequence is one of the codes with worst BER performance when the number of users is increased from 1 to 60 as shown in Figure 4 . However, the BER performance of large set Kasami code becomes better when the numbers of users increase from 60 to 64 users. This is due to the cross-correlation characteristics of the large set Kasami code sequence. i.e. when more number of users added to produce combined signal to be transmitted at the base station, the crosscorrelation value between desired user's code sequence and interfering user's code sequence decreases from -59 of 60 users to -71 of 64 . As seen in Figure 3, the BER performance of the large set Kasami code sequence significantly improves when the SNR value is increased. This implies that the receiver has to cope with both noise and MAI introduced by the channel when implementing large set Kasami code sequence in synchronous CDMA system under flat fading channel. Hence, large set Kasami code sequences have much lower signal levels at specific sampling interval as demonstrated by the eye diagram in Figure 8. On the other hand, the proposed HOLS, Walsh and orthogonal small set Kasami code sequences have much better BER performance than those of large set Kasami code sequence.

Gold code sequence, as presented in Figure 4, has one of the worst BER performances when increasing the number of users, while displaying constant BER performance when increasing the SNR value as seen in Figure 3. This implies that the noise at the receiver is mainly dominated by the MAI introduced by the channel when implementing Gold code sequence in synchronous CDMA system under flat fading channel. As can be seen in Figure 3, Gold and large set Kasami code sequences have similar BER values when SNR equals to $0 \mathrm{~dB}$. The BER performance of Gold code sequence does not improve when increasing SNR values while that of the large set Kasami code sequence does. Besides, large set Kasami code sequence has higher signal levels than Gold code sequence at specific sampling interval as shown in the eye diagrams in Figure 8 and 9. Therefore, Gold code sequence has worse BER performance than the large set Kasami code sequence.

As shown in Figure 3, by converting the nonorthogonal large set Kasami code sequence into orthogonal code sequence, the BER performance of the code sequence has been improved to the level of Walsh code sequence in synchronous CDMA system under flat fading channel condition.

\section{B. BER of HOLS code sequence under Rayleigh frequency selective fading channel condition}

As presented in Figure 10, HOLS, Walsh and orthogonal small set Kasami code sequences all have similar BER performance and they outperform the Gold and the large set Kasami code sequences under Rayleigh frequency selective fading channel in synchronous CDMA system. The BER performance of large set Kasami code sequence is slightly worse than that of Gold code sequence, and the reasons are discussed in the following paragraphs.
As can be seen in Figure 11, the Walsh code sequence has a consistent BER performance for increasing number of users under frequency selective fading channel because it has zero cross-correlation function (crosscorrelation value is zero for any chip shifts). Due to the zero cross-correlation between its code sequences, the Walsh code sequence can maintain orthogonality under frequency selective fading channel, and so there are no interferences from other users, therefore the receiver needs only to cope with the noise introduced by the channel.

The BER performance of the HOLS and orthogonal small set Kasami code sequences fluctuate slightly as seen in Figure 11, due to the characteristics of the frequency selective fading channel and non-zero CCF of the codes unlike Walsh code. When passing through the selective fading channel, each chip of a code sequence will have different attenuations for different frequencies; as a result due to non-zero $\mathrm{CCF}$, the HOLS and orthogonal small set Kasami code sequences cannot maintain the orthogonality, and hence the crosscorrelation value will slightly fluctuate when the number of users is increased. Therefore, the BER performances for these codes fluctuate. However, the BER performance of the code sequences improves when the SNR value is increased as seen in Figure 10. It means that implementing the HOLS code sequence in synchronous CDMA system under frequency selective fading channel, the receiver has to cope with noise and MAI introduced by the channel. Besides, as presented in Figure 11, the HOLS code sequence attains similar BER performance to Walsh code sequence when it reaches 64 numbers of users, since the HOLS code and Walsh code sequences have similar signal levels at specific sampling interval as can be seen in the eye diagrams in Figure 12 and Figure 13. While orthogonal small set Kasami attains similar BER performance to Walsh code when it reaches 40 numbers of users and having constant BER performance for increasing numbers of users started at 40 numbers of users. This is due to characteristic (crosscorrelation function) of orthogonal small set Kasami code sequence i.e. when more number of users added to produce combined signal to be transmitted at the base station, the cross-correlation value between desired user's code sequence and interfering user's code sequence is constant which is the same as Walsh code. In fact it has similar signal levels to that of Walsh code at specific sampling interval as shown in the eye diagrams in Figure 14. Therefore the HOLS, Walsh and orthogonal small set Kasami code sequences have similar BER performance in synchronous CDMA system under frequency selective fading channel.

The BER performance of Gold and large set Kasami code sequences, as shown in Figure 11, fluctuate more widely and are worse than that of the orthogonal code sequences for increasing number of users, however becomes better when increasing the SNR values as seen in Figure 10. This implies that the receiver has to cope with noise and MAI introduced by the channel when implementing Gold and large set Kasami code sequences in synchronous CDMA system under frequency selective fading channel. As these code sequences have lower signal levels than the orthogonal code sequences at specific sampling interval as shown in the eye diagrams 
in Figure 15 and Figure 16, Gold and large set Kasami code sequences have worse BER performance than those of orthogonal code sequences.

As presented in Figure 10, the BER performance of Gold code sequence is slightly better than that of large set Kasami code sequence, as Gold code sequence has slightly higher signal levels at specific sampling interval than that of large set Kasami code sequence as seen in Figure 15 and Figure 16.

Therefore, as shown in Figure 10, the HOLS code sequence is shown to reach that of Walsh code sequence in BER performance in synchronous CDMA system under frequency selective fading channel condition.

\section{Cross-correlation property of HOLS code sequence}

Gold and large set Kasami code sequences as presented in Figure 17 and Figure 18, have crosscorrelation value between two different code sequences in a family chip set is non-zero at zero time shifts and across all the time shifts. It means that these code sequences do not have zero multiple access interference (MAI) and hence, their performance will be limited by the MAI or both MAI and the noise introduced by the channel as shown in Figure 3, 4, 10, and 11.

As seen in Figure 19, Walsh code sequence has zero cross-correlation value at any time shift which means that the code sequence does not suffer from MAI. Therefore, the performance of Walsh code sequence can be considered to be noise limited channel rather than the multiple access interference (MAI) from other users as shown in Figure 3, 4, 10, and 11.

The proposed HOLS code sequence, and as well as the orthogonal small set Kasami code sequence have only zero cross-correlation values at zero time shifts and at some non-zero time shifts as shown in Figure 20 and Figure 21. Therefore, the HOLS and orthogonal small set Kasami code sequences do not possess zero multiple access interference and so the performance of HOLS and orthogonal small set Kasami code sequences will be limited by both multiple access interference (MAI) and the noise introduced by the channel as shown in Figure 10 and 11 .

\section{Capacity of the HOLS and other selected spreading codes}

For large set Kasami code sequence of value, $n=2$ $\bmod 4$ the minimum number of registers $n$ is 6 and the sequence length $\mathrm{N}$ is 63 chips generated. Referring to equations (2) and (3), the number of groups and number of members in each group can generate is 249 and 64 respectively.

Therefore, the family size of HOLS code sequence that can be generated using (1) is 15936 code sequences. This means, in terms of number of users (or receiver nodes) that can be accommodated in a CDMA system, the HOLS code sequence can accommodate 249 groups of users where each group contains 64 users. These values are tabulated in Table I and compared with those of other selected spreading code sequences. As shown in Table I, the HOLS code sequence has the largest code set.

In terms of computational savings at the receiver, it was shown that Walsh orthogonal code sequence can be implemented on [12]. Therefore, the proposed HOLS code sequence can also be implemented on that work since each member in a code group is orthogonal to each other like Walsh code sequence.

\section{E. Auto-correlation property of HOLS code sequence}

Auto-correlation property is an important factor in synchronization at the receiver in synchronous CDMA system. The point in time, when the receiver can detect sufficiently high signal strength indicates that the starting point of the code is found.

Gold, large set Kasami and orthogonal small Kasami code sequences have peak values at zero time shifts that are much higher than the side lobes as presented in Figure 22, 23 and 24 respectively. Because of these features, Gold, large set Kasami and orthogonal small Kasami code sequences can easily detect the starting point of their code sequences. Therefore, Gold, large set Kasami and orthogonal small set Kasami code sequences can be categorized as code sequences having a good $\mathrm{ACF}$ in terms of synchronization at the receiver.

As seen in Figure 25, the proposed HOLS code sequence has similar ACF to those of Gold, large set Kasami and orthogonal small set Kasami code sequences i.e. having the peak value at zero time shifts much higher than the side lobes and so has very good synchronization at the receiver. Therefore the proposed HOLS code sequence is considered to have very good synchronization at the receiver.

However, as shown in Figure 26, the peak value of Walsh code sequence at zero time shifts is exactly the same as the side lobe values. Therefore, it is not possible to detect the starting point of the Walsh code sequence at the receiver side when the Walsh code sequences are not synchronized. Hence, Walsh code sequence displays an ACF much worse than Gold, large set Kasami, orthogonal small set Kasami and HOLS code sequences.

\section{CONCLUSION}

In this paper, the design of hybrid orthogonal large set (HOLS) code sequence has been proposed and evaluated. The proposed code performance has been shown to be superior to the existing non-orthogonal code sequences while being comparable to Walsh and orthogonal small set Kasami code sequences, and yet supporting largest cell-based synchronous CDMA system. The salient features of the HOLS code sequence and its performance are summarized below.

a. The HOLS code sequence possesses comparable CCF (cross-correlation function) with Walsh, and orthogonal small set Kasami code sequences in terms of MAI (multiple access interference) under flat fading channel which has zero MAI. While the proposed orthogonal code sequence has a much better CCF than that of Gold and large set Kasami code sequences, since these code sequences have worse BER performance for increasing number of users.

b. In terms of MAI, under frequency selective fading channel, CCF of the HOLS code sequence is worse than that of Walsh code sequence. While the HOLS code sequence has comparable $\mathrm{CCF}$ with orthogonal small set Kasami code sequence. It is because the HOLS and orthogonal small set Kasami code sequences do not possess zero MAI for increasing number of users while Walsh code sequence does. 
c. ACF of the HOLS code sequence is impulsive (the main lobe is much higher than the side lobes) that is comparable to those of Gold, large set Kasami and orthogonal small set Kasami code sequences and much better than that of Walsh code sequence.

d. The BER performance of the HOLS code sequence is the same as that of Walsh and orthogonal small set Kasami code sequences under both flat and frequency selective fading channels in synchronous CDMA system and outperforms Gold and large set Kasami code sequences.

e. Capacity of the HOLS code sequence for code length of 64 chips (15936 code sequences) outperforms Walsh, orthogonal small set Kasami, Gold and large set Kasami code sequences.

\section{ACKNOWLEDGEMENT}

We would like to thank the Indonesian Government (Dikti) for funding this research work. We also thank the RSO, LJMU for funding part of the conference attendance.

\section{REFERENCES}

[1]. Hoshyar, R., Wathan, F.P., and Tafazolli, R. (2008) 'Novel lowdensity signature for synchronous CDMA systems over AWGN channel', IEEE Transactions on Signal Processing, Vol. 56, pp.1616-1626

[2]. Cheng, H., Ma, M. and Jiao, B. (2009) 'On the design of comb spectrum code for multiple access scheme', IEEE Transactions on Communications, Vol. 57, No. 3, pp.754-763

[3]. Nikjah, R. and Beaulieu, N.C. (2008) 'On antijamming in general CDMA systems-Part I: Multiuser capacity analysis',
IEEE Transactions on Wireless Communications, Vol. 7, No. 5, pp. 1646-1655

[4]. Jatunov, L. and Madisetti, V.K. (2006) 'Computationallyefficient SNR estimation for band limited wideband CDMA systems', IEEE Transaction on Wireless Communications, Vol. 5, pp.3480-3491

[5]. Van Houtum, W.J. (2001) 'Quasi-synchronous code-division multiple access with high-order modulation', IEEE Transactions on Communications, Vol. 49, No. 7, pp.1240-1249

[6]. Adeola, F.A., Adekunle, A.S. and Peter, A.O. (2011) 'Direct sequence CDMA system using finite-time altered sinusoidal signals as orthogonal signatures', IEEE 3rd International Conference on Adaptive Science and Technology (ICAST 2011), pp. $174-177$

[7]. Pal, M. and Chattopadhyay, S. (2010) 'A novel orthogonal minimum cross-correlation spreading code in CDMA system', IEEE International Conference on Emerging Trends in Robotics and Communication Technologies, pp.80-84

[8]. Akansu, A.N. and Poluri, R. (2007) 'Walsh-like nonlinear phase orthogonal codes for direct sequence CDMA communications', IEEE Transactions on Signal Processing, Vol. 55, No. 7, pp. 3800-3806

[9]. Akansu, A.N. and Poluri, R. (2007) 'Design and performance of spread spectrum Karhunen-Loeve transform for direct sequence CDMA communications', IEEE Signal Processing Letters, Vol. 14, No. 12, pp.900-903

[10]. Kedia, D., Duhan, M. and Maskara, S.L. (2010) 'Evaluation correlation properties of orthogonal spreading codes for CDMA wireless mobile communication', IEEE International Advance Computing Conference (IACC), pp.325-330

[11]. Xiangyong, Z., Liu, J.Q., and Lei, H. (2007) 'Generalized Kasami sequences: The large set', IEEE Transactions on Information Theory, Vol. 53, pp.2587-2598

[12]. X. Wang and H. Ge, "Spreading codes enabling warp converging Wiener filters for multiuser detection in CDMA Systems," in Proc. IEEE Int. Conf. Commun., Beijing, China, May 2008.

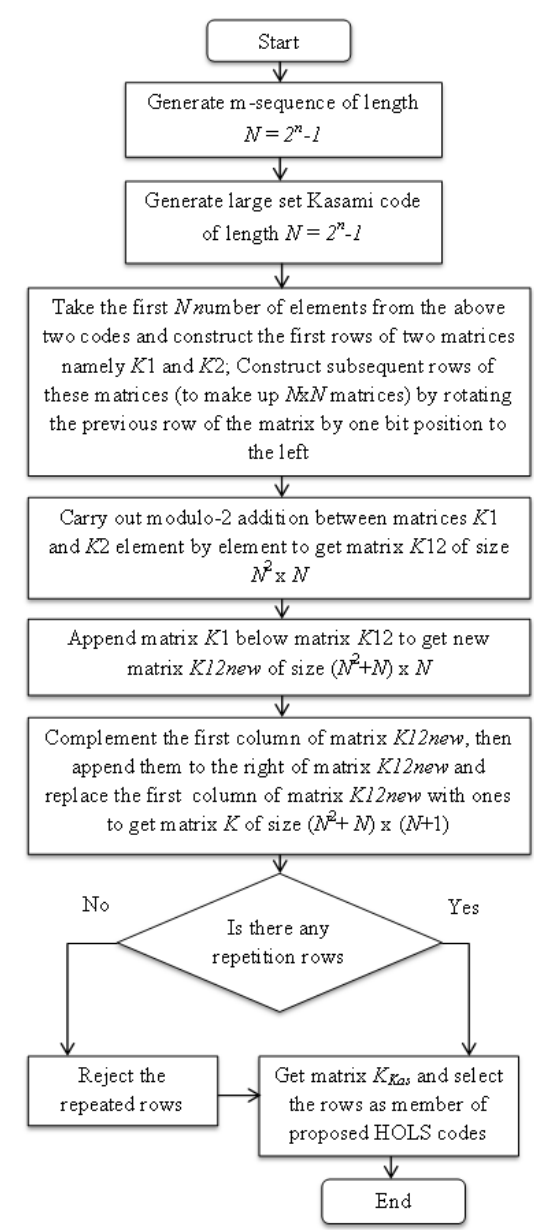

Figure 1. Algorithms for the design of the HOLS code sequence 


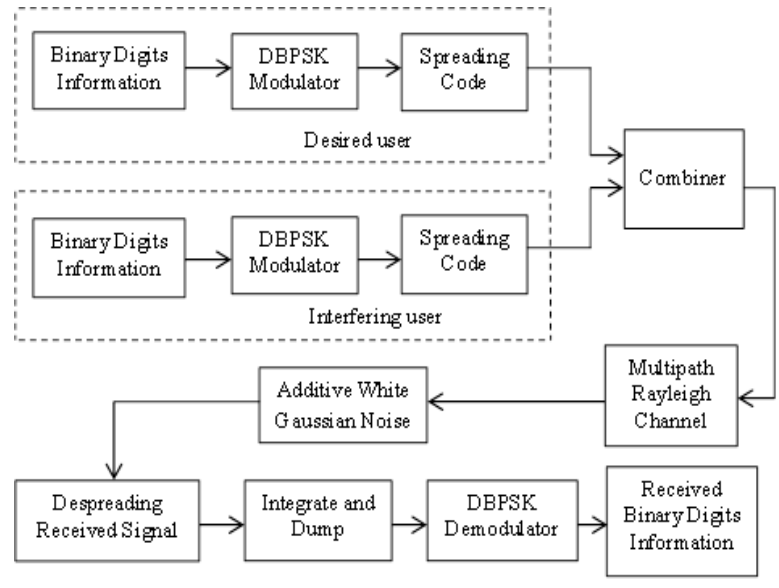

Figure 2. Block diagram for the simulation of synchronous CDMA system

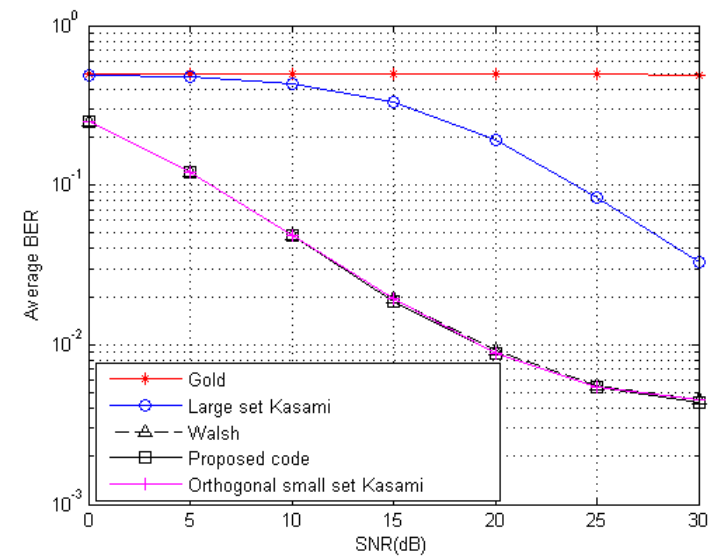

Figure 3. BER performance of the spreading codes over Rayleigh flat fading channel

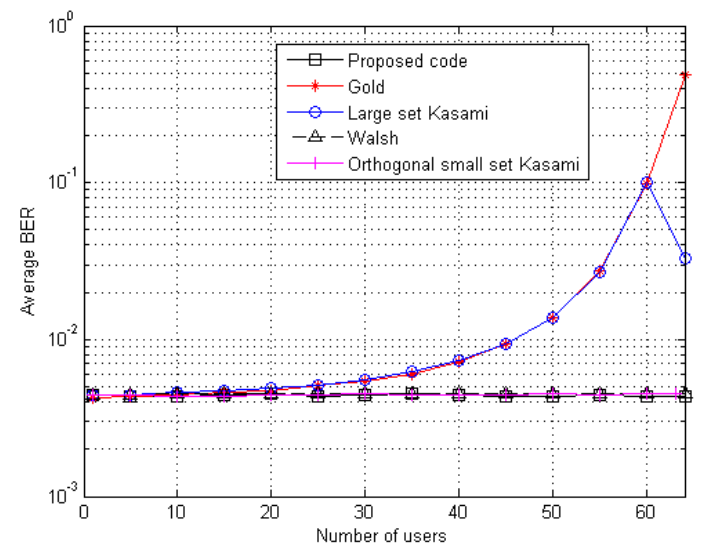

Figure 4. BER performance of the spreading codes as function of number of users over Rayleigh flat fading channel for SNR $=30 \mathrm{~dB}$

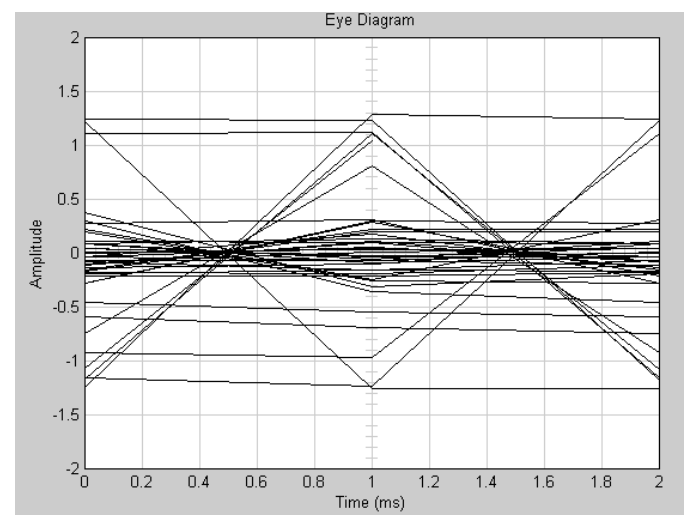

Figure 5. Eye diagram of HOLS code sequence for $\mathrm{SNR}=30 \mathrm{~dB}$

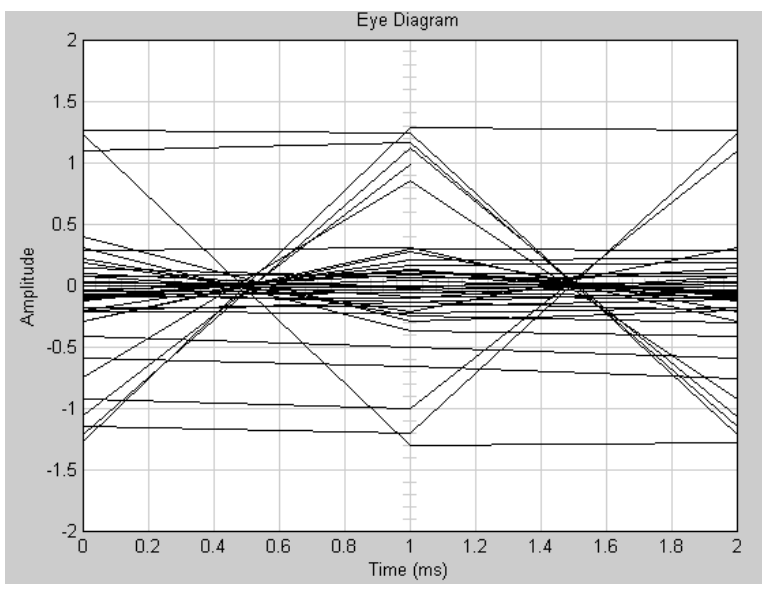

Figure 6. Eye diagram of Walsh code sequence for $\mathrm{SNR}=30 \mathrm{~dB}$

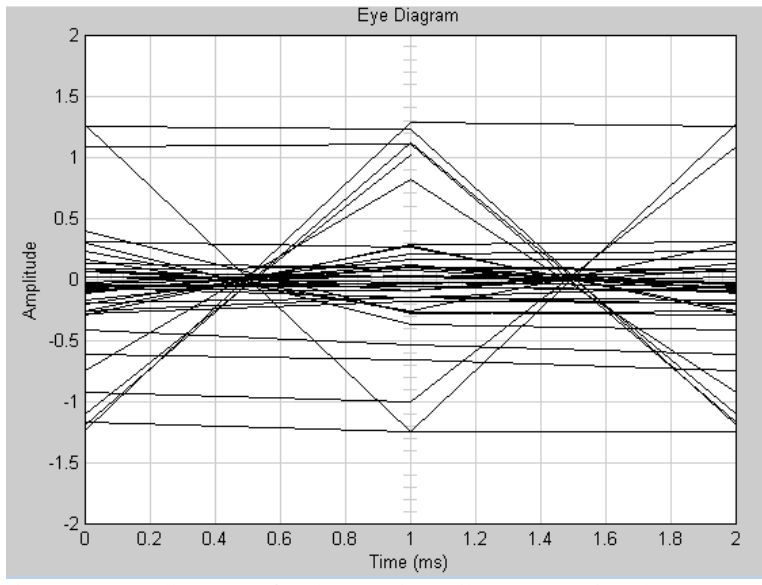

Figure 7. Eye diagram of orthogonal small set Kasami code sequence for $\mathrm{SNR}=30 \mathrm{~dB}$

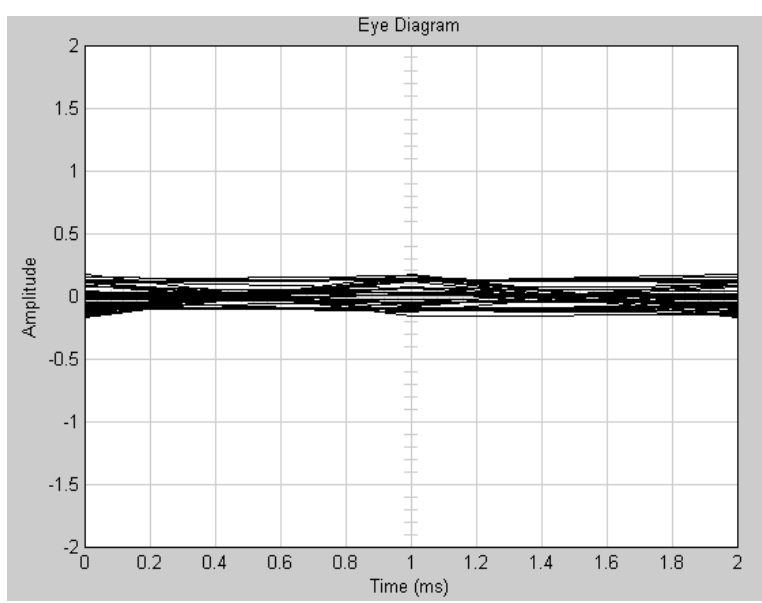

Figure 8. Eye diagram of large set Kasami code sequence for $\mathrm{SNR}=30 \mathrm{~dB}$

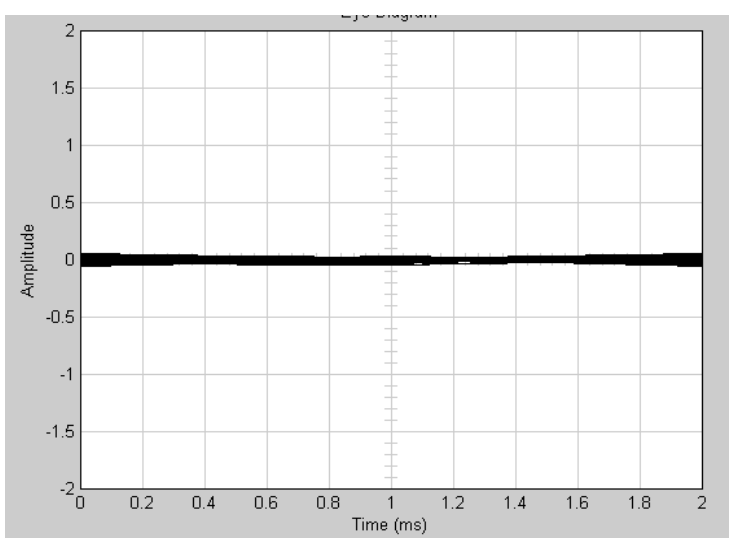

Figure 9. Eye diagram of Gold code sequence for $\mathrm{SNR}=30 \mathrm{~dB}$ 


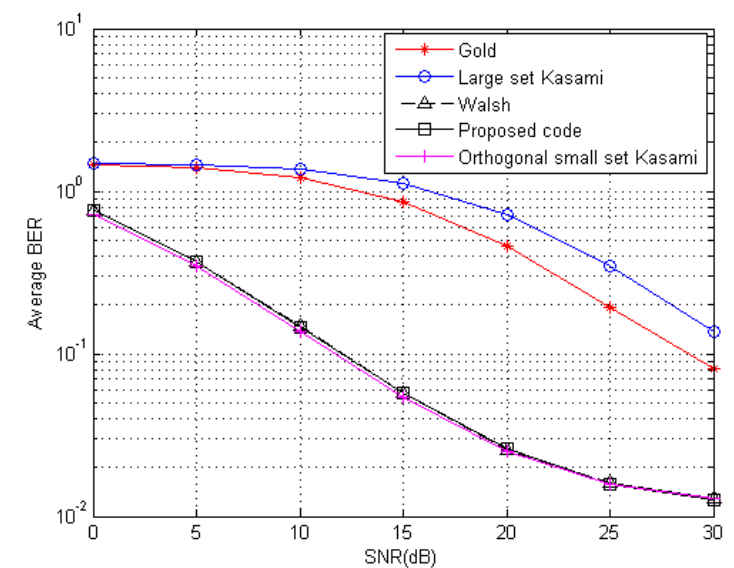

Figure 10. BER performance of the spreading codes over Rayleigh frequency selective fading channel

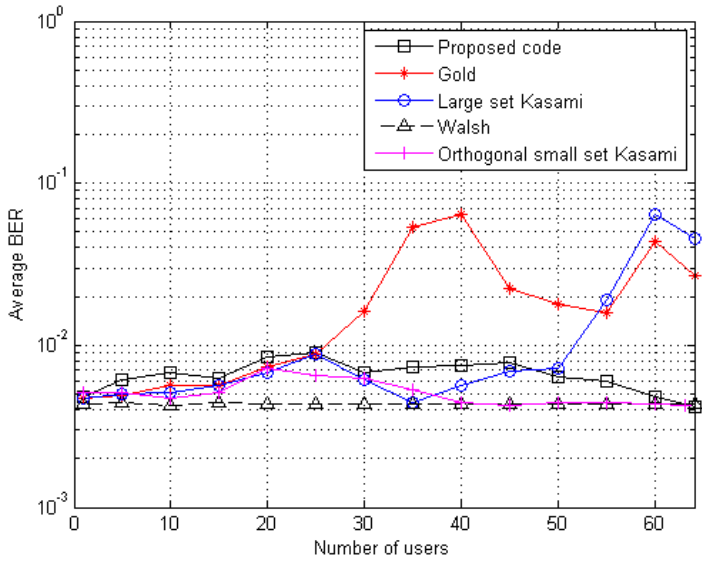

Figure 11. BER performance of the spreading codes as function of number of users over Rayleigh frequency selective fading channel for $\mathrm{SNR}=30 \mathrm{~dB}$

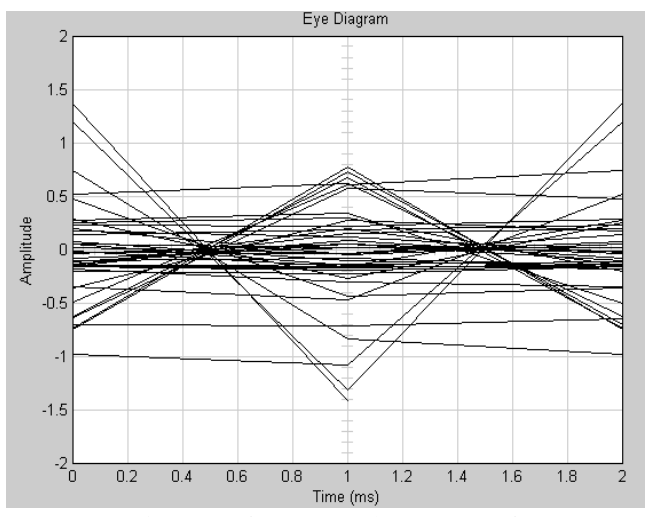

Figure 12. Eye Diagram of HOLS code sequence for $\mathrm{SNR}=30 \mathrm{~dB}$

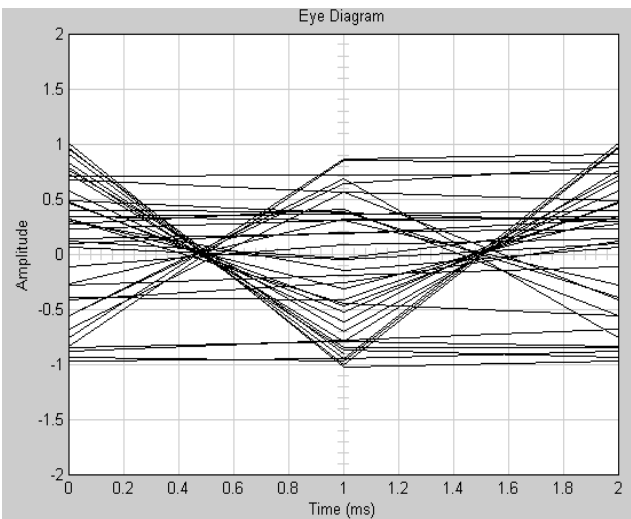

Figure 13. Eye Diagram of Walsh code sequence for SNR $=30 \mathrm{~dB}$

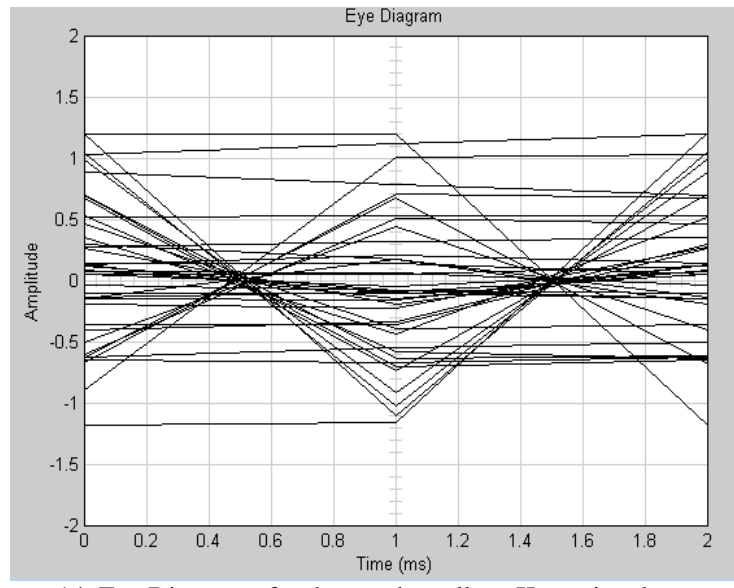

Figure 14. Eye Diagram of orthogonal small set Kasami code sequence for $\mathrm{SNR}=30 \mathrm{~dB}$

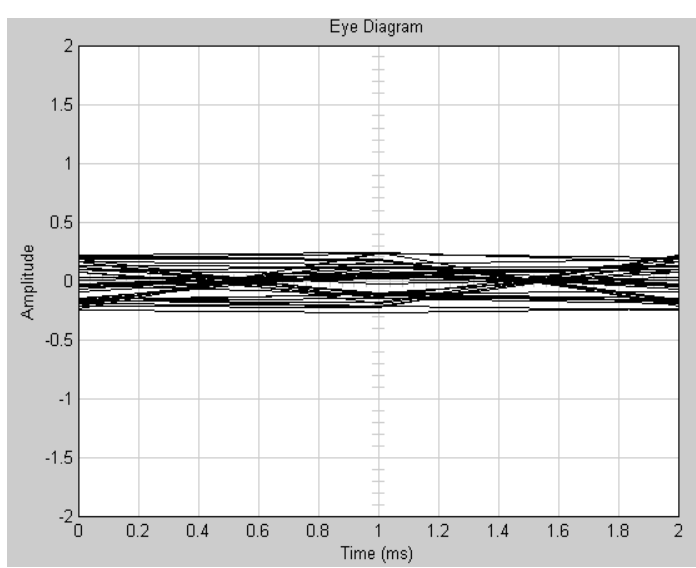

Figure 15. Eye Diagram of Gold code sequence for $\mathrm{SNR}=30 \mathrm{~dB}$

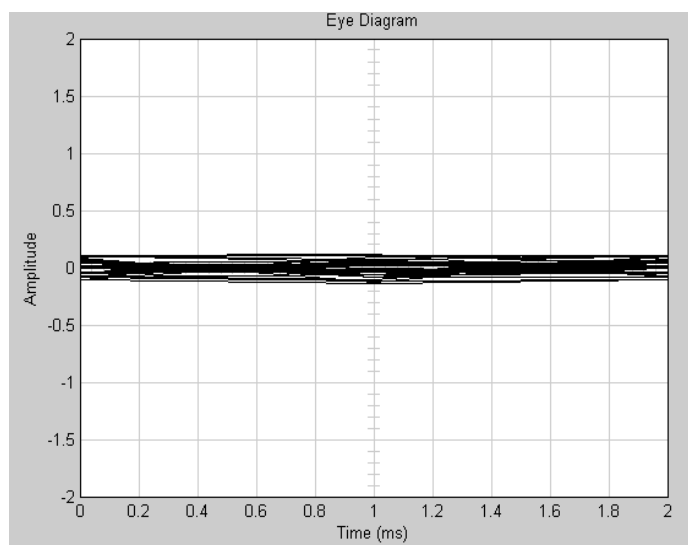

Figure 16. Eye Diagram of large set Kasami code sequence for $\mathrm{SNR}=30 \mathrm{~dB}$

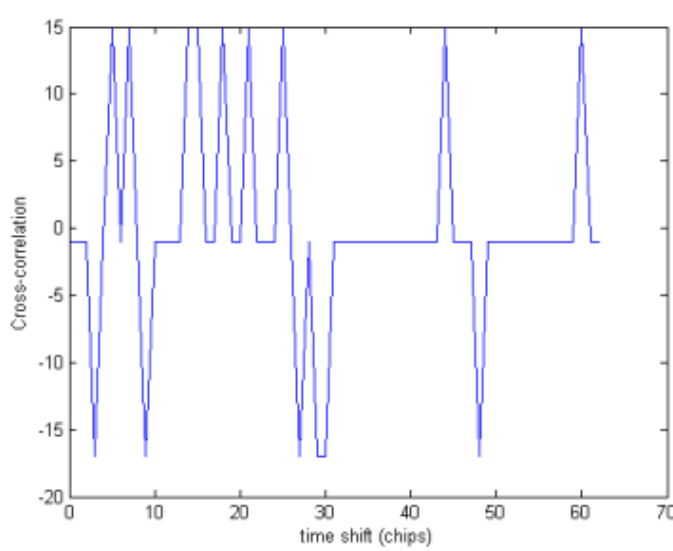

Figure 17. Cross-correlation function of Gold code sequence 


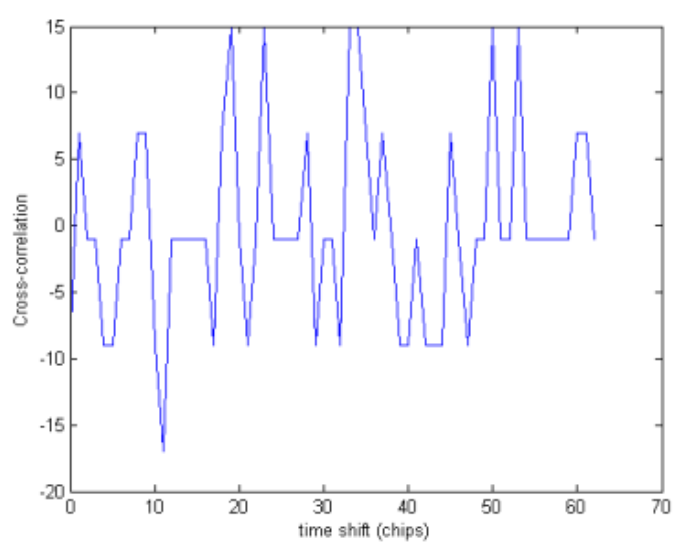

Figure 18. Cross-correlation function of large set Kasami code sequence

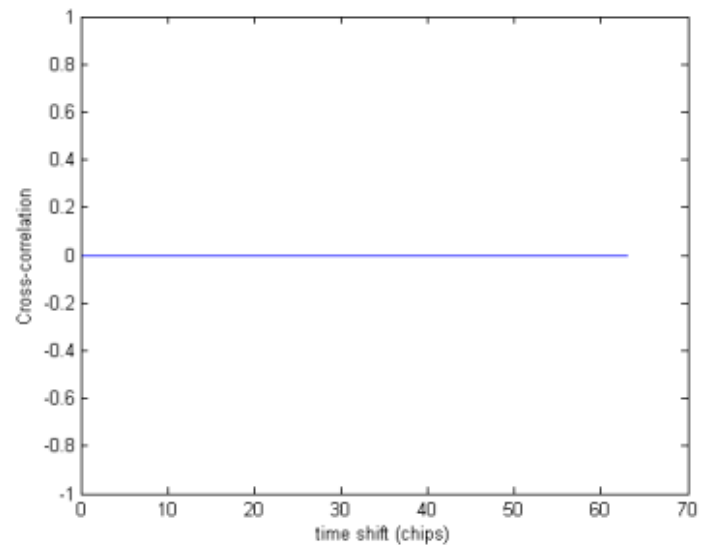

Figure 19. Cross-correlation function of Walsh code sequence

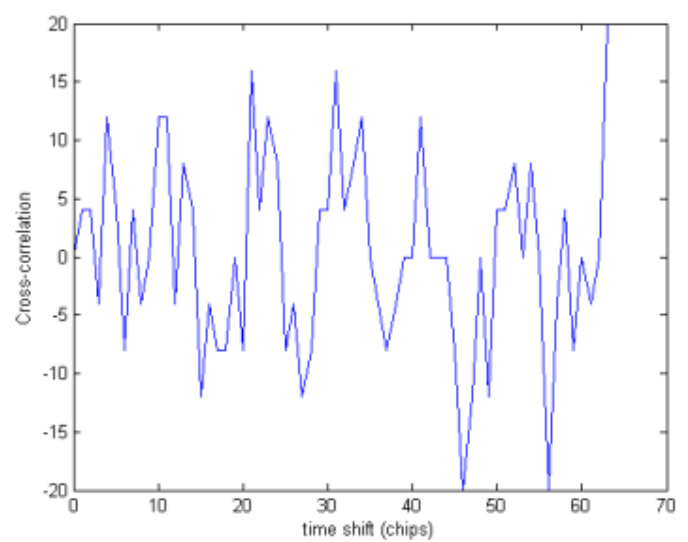

Figure 20. Cross-correlation function of HOLS code sequence

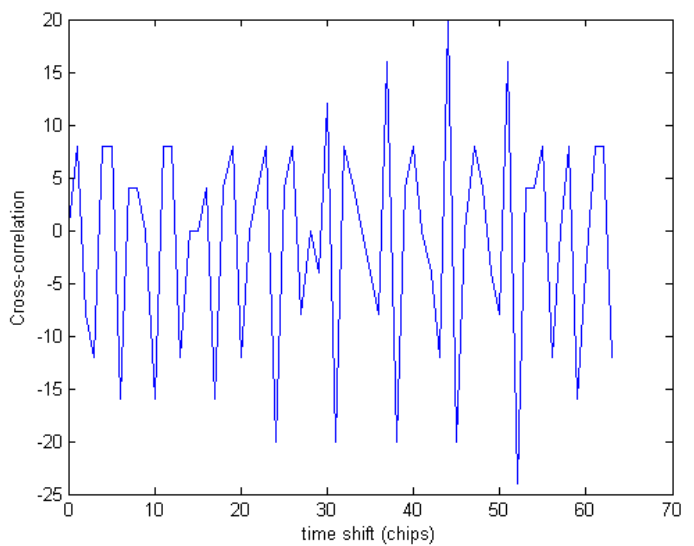

Figure 21. Cross-correlation function of orthogonal small set Kasami code sequence

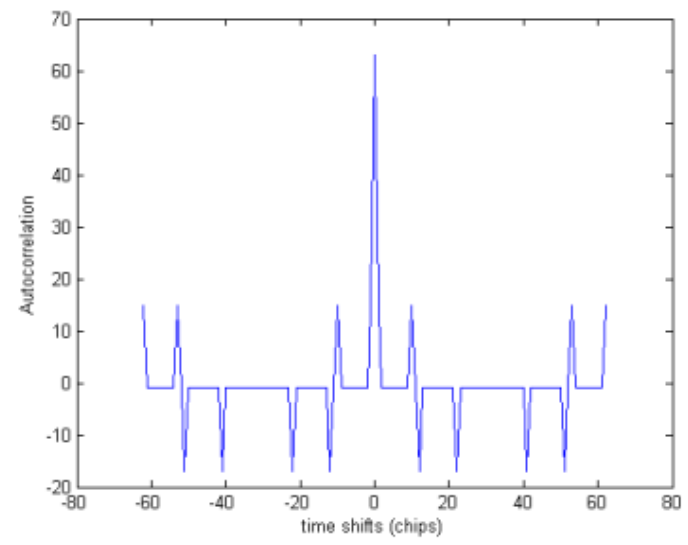

Figure 22. Auto-correlation function of Gold code sequence

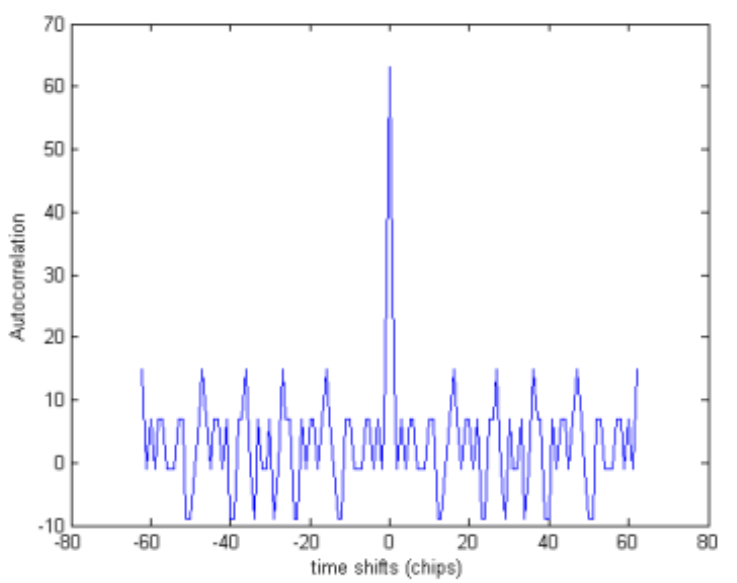

Figure 23. Auto-correlation function of large set Kasami code sequence

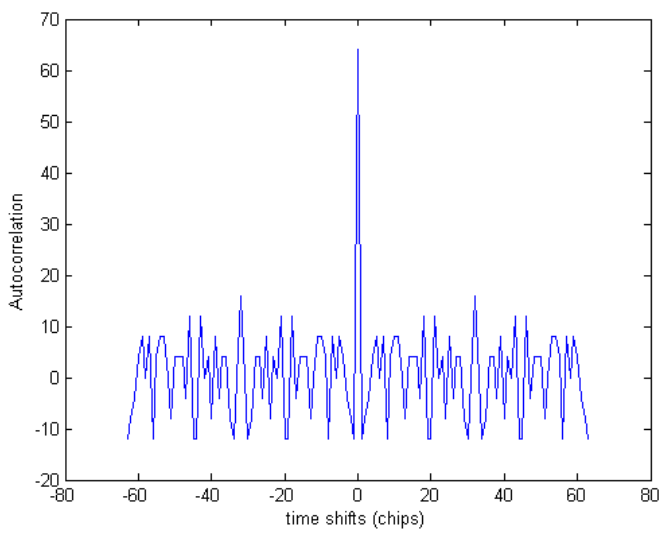

Figure 24. Auto-correlation function of orthogonal small set Kasami code sequence

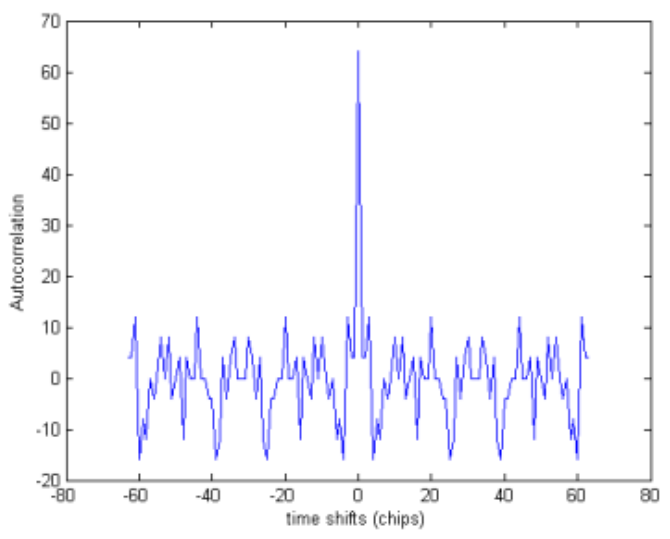

Figure 25. Auto-correlation function of HOLS code sequence 


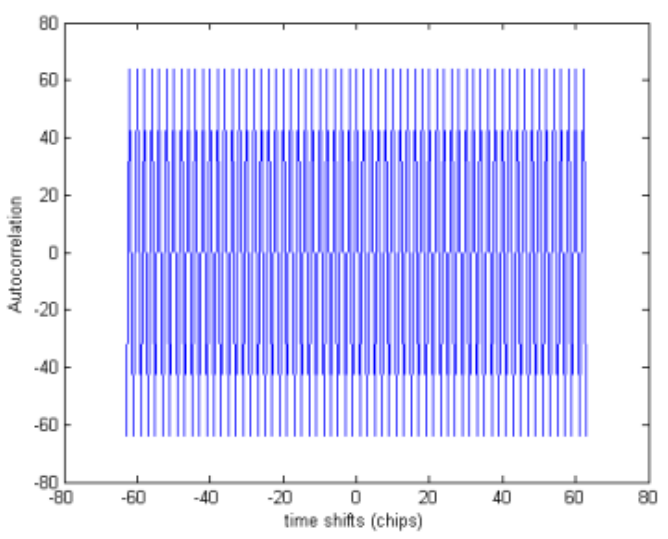

Figure 26. Auto-correlation function of Walsh code sequence
TABEL I

CAPACITY OF THE HOLS AND SELECTED SPREADING CODE SEQUENCES

\begin{tabular}{cccc}
\hline \hline $\begin{array}{c}\text { Spreading } \\
\text { code sequence }\end{array}$ & $\begin{array}{c}\text { Length of } \\
\text { code } \\
\text { sequence }\end{array}$ & $\begin{array}{c}\text { Number of } \\
\text { groups }\end{array}$ & $\begin{array}{c}\text { Capacity of the } \\
\text { synchronous CDMA } \\
\text { system implementing } \\
\text { the code }\end{array}$ \\
\hline Gold & 63 chips & 1 & 65 codes \\
$\begin{array}{c}\text { Large set } \\
\text { Kasami }\end{array}$ & 63 chips & 1 & 520 codes \\
Walsh & 64 chips & 1 & 64 codes \\
HOLS & 64 chips & 249 & 15936 codes \\
$\begin{array}{c}\text { Orthogonal } \\
\text { Small set } \\
\text { Kasami }\end{array}$ & 64 chips & 8 & 504 codes \\
\hline \hline
\end{tabular}

